\title{
Super-Spatial Structure Prediction Compression of Medical Image Sequences
}

\author{
M Ferni Ukrit*1, GR Suresh ${ }^{2}$ \\ ${ }^{1}$ Department of CSE, Sathyabama University, Chennai, Tamilnadu, India \\ ${ }^{2}$ Department of ECE, Easwari Engineering College, Chennai, Tamilnadu, India \\ *Corresponding author, e-mail: fernijegan@gmail.com
}

\begin{abstract}
The demand to preserve raw image data for further processing has been increased with the hasty growth of digital technology. In medical industry the images are generally in the form of sequences which are much correlated. These images are very important and hence lossless compression Technique is required to reduce the number of bits to store these image sequences and take less time to transmit over the network The proposed compression method combines Super-Spatial Structure Prediction with interframe coding that includes Motion Estimation and Motion Compensation to achieve higher compression ratio. Motion Estimation and Motion Compensation is made with the fast block-matching process Inverse Diamond Search method. To enhance the compression ratio we propose a new scheme Bose, Chaudhuri and Hocquenghem $(\mathrm{BCH})$. Results are compared in terms of compression ratio and Bits per pixel to the prior arts. Experimental results of our proposed algorithm for medical image sequences achieve 30\% more reduction than the other state-of-the-art lossless image compression methods.
\end{abstract}

Keywords: Lossless Compression, Medical Image Sequences, Super-Spatial Structure Prediction, Interframe Coding, MEMC

\section{Introduction}

Medical Science grows very fast and hence each hospital, various medical organizations needs to store high volume of digital me dical image sequences that includes Computed Tomography (CT), Magnetic Resonance Image (MRI), Ultrasound and Capsule Endoscope (CE) images. As a result hospitals and medical organizations have high volume of images with them and require huge disk space and transmission bandwidth to store this image sequences [1].The solution to this problem could be the application of compression. Image compression techniques reduce the number of bits required to represent an image by taking advantage of coding, inter-pixel and psycho visual redundancies. Medical image compression is very important in the present world for efficient archiving and transmission of images [2]. Image compression can be classified as lossy and lossless. Medical imaging does not require lossy compression due to the following reason. The first reason is the incorrect diagnosis due to the loss of useful information. The second reason is the operations like image enhancement may emphasize the degradations caused by lossy compression. Lossy scheme seems to be irreversible. But lossless scheme is reversible and this represents an image signed with the smallest possible number of bits without loss of any information thereby speeding up transmission and minimizing storage requirement. Lossless compression reproduces the exact replica of the original image without any quality loss [3]. Hence efficient lossless compression methods are required for medical images [4]. Lossless compression includes Discrete Cosine Transform, Wavelet Compression [5], Fractal Compression, Vector Quantization and Linear Predictive Coding. Lossless consist of two distinct and independent components called modeling and coding. The modeling generates a statistical model for the input data. The coding maps the input data to bit strings [6].

Several Lossless image compression algorithms were evaluated for compressing medical images. There are several lossless image compression algorithms like Lossless JPEG,JPEG 2000,PNG,CALIC and JPEG-LS.JPEG-LS has excellent coding and best possible compression efficiency [1]. But the Super-Spatial Structure Prediction algorithm proposed in [7] has outperformed the JPEG-LS algorithm. This algorithm divides the image into two regions, structure regions (SRs) and non-structure regions (NSRs). The structure regions are encoded with Super-Spatial Structure Prediction technique and non-structure regions are encoded using 
CALIC. The idea of Super-Spatial Structure Prediction is taken from video coding. There are many structures in a single image. These include edges, pattern and textures. This has relatively high computational efficiency. No codebook is required in this compression scheme because the structure components are searched within the encoded image regions [8]. CALIC is a spatial prediction based scheme which uses both context and prediction of the pixel values [9] which accomplishes relatively low time and space complexities. A continuous tone mode of CALIC includes the four components, prediction, context selection and quantization, context modeling of prediction errors and entropy coding of prediction error [10].

Most of the lossless image compression algorithms take only a single image independently without utilizing the correlation among the sequence of frames of MRI or CE images. Since there is too much correlation among the medical image sequences, we can achieve a higher compression ratio using inter-frame coding. The idea of compressing sequence of images was first adopted in [11] for lossless image compression and was used in [12], [13], [14] for lossless video compression. The Compression Ratio (CR) was significantly low (i.e.) 2.5 which was not satisfactory. Hence in [1] they have combined JPEG-LS with interframe coding to find the correlation among image sequences and the obtained ratio was 4.8.Super-Spatial Structure Prediction algorithm proposed in [15] has outperformed JPEG-LS. However this ratio can be enhanced using Super-Spatial Structure Prediction technique and Bose, Chaudhuri and Hocquenghem (BCH).Super-Spatial Structure Prediction is applied with a fast block matching algorithm Inverse Diamond Search (IDS) algorithm which include lower number of searches and search points [16]. BCH scheme is used repeatedly to increase the compression ratio [17].

In this paper, we propose a hybrid algorithm for medical image sequences. The proposed algorithm combines Super-Spatial Structure Prediction technique with inter-frame coding and a new innovative scheme $\mathrm{BCH}$ to achieve a high mpression ratio. The Compression Ratio (CR) can be calculated by the equation (1) and PSNR by equation (2)

$$
\begin{aligned}
& \mathrm{CR}=\text { Original Image Size/Compressed Image Size } \\
& \text { PSNR }=20 * \log 10 \text {. }
\end{aligned}
$$

This paper is organized as follows:

Section II explains the methodology used which includes Overview, Super-Spatial Structure Prediction, Motion Estimation and Motion Compensation, Motion Vector, Block Matching Algorithm and $\mathrm{BCH}$. Section III discusses the results obtained for the proposed methodology.

\section{Research Method}

\subsection{Overview}

The objective of the proposed method is to enhance the compression efficiency using Super-Spatial Structure Prediction (SSP) technique combined with Motion Estimation and Motion Compensation (MEMC). The compression ratio is further enhanced by $\mathrm{BCH}$ algorithm an error correcting technique. Figure1 illustrates the complete encoding technique of the proposed method. The steps in the proposed method are discussed.

Step 1: Given an image sequence, input the first image to be compressed

Step 2: The image is classified as Structure Regions (SRs) and Non-Structure Regions (NSRs). SRs are encoded using SSP and NSRs are encoded using CALIC

Step 3: The first image will be compressed by Super-spatial Structure Prediction since there is no reference frame.

Step 4: Now the second frame becomes the current frame and the first frame becomes the reference frame for the second frame.

Step 5: Inter-frame coding includes MEMC process to remove temporal redundancy. Intercoded frame will be divided to blocks known as macro blocks.

Step 6: The encoder will try to find a similar block as the previously encoded frame. This process is done by a block matching algorithm called Inverse Diamond Search.

Step 7: If the encoder succeeds on its search the block is directly encoded by a vector known as Motion Vector. 
Step 8: After MEMC is done the difference of images is processed for compression. The difference is also compressed using SSP compression. MV derived from MEMC is also compressed.

Step 9: BCH converts SSP output code to binary and divide it to 7 bits each

Step 10: Each block is checked if it is valid codeword or not.BCH converts the valid block to 4 bits.

Step 11: This method adds 1 as an indicator for the valid codeword to an extra file called map otherwise if it is not a codeword it remains 7 and adds 0 to the same file.

Step 12: This step is iterated three times to improve CR.

Step 13: Flag bits and the encoded bits are concatenated.

Step 14: Once the compression of the second frame is done it becomes the reference frame for the third frame and this processing will be repeated for the next image until the end of image sequence

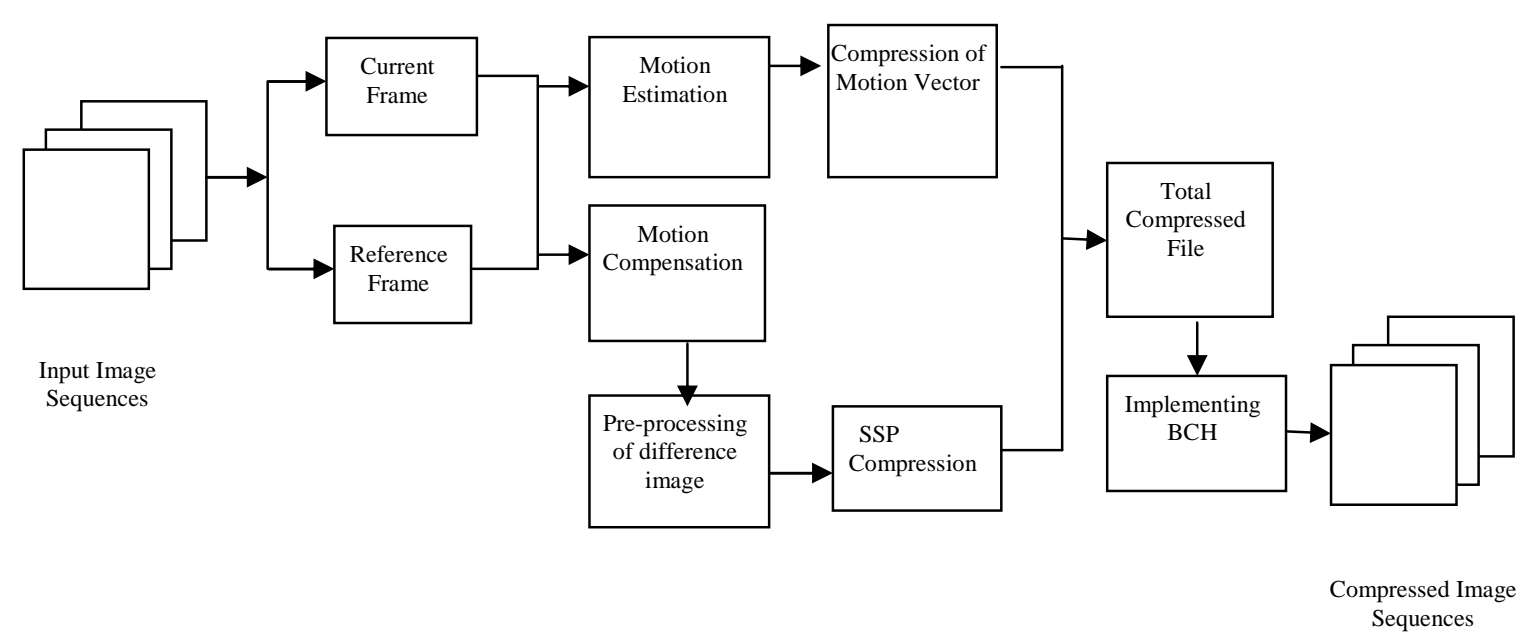

Figure 1. Encoding Technique of the Proposed Method

\subsection{Super-Spatial Structure Prediction}

Super-Spatial Structure Prediction borrows its idea from motion prediction [18].In SSP an area is searched within the previously encoded image region to find the prediction of an image block. The reference block that results in the minimum block difference is selected as the optimal prediction. Sum of Absolute Difference (SAD) is used to measure the block difference. The size of the prediction unit is an important parameter. When the size is small the amount of prediction and coding overhead will become large. If larger prediction unit is used the overall prediction efficiency will decrease. In this paper, a good substitution between this two is proposed. The image is partitioned into blocks of $4 \times 4$ and classifies these blocks to structure and non-structure blocks. Structure blocks are encoded using SSP and non-structure blocks using CALIC.

CALIC is a spatial prediction based scheme in which GAP (Gradient Adjusted Predictor) is used for adaptive image prediction. The image is classified to SRs and NSRs and then SSP is applied to SRs since its prediction gain in the non structure smooth regions will be very limited. This will reduce the overall computational complexity [7].

\subsection{Motion Estimation and Motion Compensation}

Motion estimation is the estimation of the displacement of image structures from one frame to another in a time sequence of $2 \mathrm{D}$ images

The steps in MEMC is stated as

- Find displacement vector of a pixel or a set of pixels between frame

- Via displacement vector, predict counterpart in present frame

- Prediction error, positions, motion vectors are coded \& transmitted 
Motion estimation can be very computationally intensive and so this compression performance may be at the expense of high computational complexity. The motion estimation creates a model by modifying one or more reference frames to match the current frame as closely as possible. The current frame is motion compensated by subtracting the model from the frame to produce a motion-compensated residual frame. This is coded and transmitted, along with the information required for the decoder to recreate the model (typically a set of motion vectors).At the same time, the encoded residual is decoded and added to the model to reconstruct a decoded copy of the current frame (which may not be identical to the original frame because of coding losses). This reconstructed frame is stored to be used as reference frame for further predictions. The inter-frame coding should include MEMC process to remove temporal redundancy. Difference coding or conditional replenishment is a very simple interframe compression process during which each frame of a sequence is compared with its predecessor and only pixels that have changes are updated. Only a fraction of pixel values are transmitted. An inter-coded frame will finitely be divided into blocks known as macro blocks. After that, instead of directly encoding the raw pixel values for each block, as it would be done for an intra-frame, the encoder will try to find a similar block to the one it is encoding on a previously encoded frame, referred to as reference frame. This process is done by a block matching algorithm. [16]. If the encoder succeeds on its search, the block could be directly encoded by a vector known as motion vector, which points to the position of the matching block at the reference frame.

\subsection{Motion Vector}

Motion estimation is using a reference frame in a video, dividing it in blocks and figuring out where the blocks have moved in the next frame using motion vectors pointing from the initial block location in the reference frame to the final block location in the next frame. For MV calculation we use Block matching algorithm as it is simple and effective. It uses Mean Square Error (MSE) for finding the best possible match for the reference frame block in the target frame. Motion vector is the key element in motion estimation process. It is used to represent a macro block in a picture based on the position of this macro block in another picture called the reference picture. In video editing, motion vectors are used to compress video by storing the changes to an image from one frame to next. When motion vector is applied to an image, we can synthesize the next image called motion compensation [11], [16]. This is used to compress video by storing the changes to an image from one frame to next frame. To improve the quality of the compressed medical image sequence, motion vector sharing is used [14].

\subsection{Block Matching}

In the block-matching technique, each current frame is divided into equal-size blocks, called source blocks. Each source block is associated with a search region in the reference frame.The objective of block-matching is to find a candidate block in the search region best matched to the source block. The relative distances between a source block and its candidate blocks are called motion vectors. Figure 3 illustrates the Block-Matching technique.

The block-matching process during the function MEMC taken from [1] takes much time hence we need a fast searching method and we have taken Inverse Diamond Search (IDS) method [16] which is the best among methods both in accuracy and speed. In the matching process, it is assumed that pixels belonging to the block are displaced with the same amount. Matching is performed by either maximizing the cross correlation function or minimizing an error criterion.

In the matching process, it is assumed that pixels belonging to the block are displaced with the same amount. Matching is performed by either maximizing the cross correlation function or minimizing an error criterion. The most commonly used error criteria are the Mean Square Error (MSE) as stated in equation (3) and the Minimum Absolute Difference (MAD) as stated in equation (4)

$$
\left.M S E=\frac{1}{M *} \sum_{i=0}^{m} \sum_{j=0}^{n}{ }^{M} M{ }_{1}(m, n)-M 2(m, n)\right]^{2}
$$

Super-Spatial Structure Prediction Compression of Medical Image Sequences (M Ferni Ukrit) 


$$
M A D=\frac{1}{M *}-\bar{N} \sum_{i=0}^{n} \sum_{j=0}{ }_{1 M}{ }_{1}(m, n)-M 2(m, n) \mid
$$

The IDS algorithm is based on MV distribution of real world video sequences. It employs two search patterns, Small Diamond Shape Pattern (SDSP) and Large Diamond Shape Pattern (LDSP) In order to reduce the number of search points, use Small Diamond Search Pattern (SDSP) as the primary shape. The entire process is discussed here.

Step 1: It first uses small diamond search pattern (SDSP) and checks five checking points to form a diamond shape.

Step 2: The second pattern consists of nine checking points and forms a large diamond shape pattern (LDSP).

Step 3: The search starts with the SDSP and is used repeatedly until the Minimum Block Distortion Measure (MBD) point lies on the search centre.

Step 4: The search pattern is then switched to LDSP.

Step 5: The position yielding minimum error point is taken as the final MV.

IDS are an outstanding algorithm adopted by MPEG-4 verification model (VM) due to its superiority to other methods in the class of fixed search pattern algorithms.

\subsection{Bose, Chaudhuri and Hocquenghem}

The binary input image is firstly divided into blocks of size 7 bits each, 7 bits are used to represent each byte and the eighth bits represent sign of the number(most significant bit).BCH checks each block if it is a valid codeword and converts the valid code word to 4 bits. It adds 1 as an indicator for the valid code word to an extra file called map. If it is not a valid code word it remains 7 and adds 0 to the same file. The map is a key for decompression to distinguish between the compressed blocks and non compressed blocks. $\mathrm{BCH}$ is repeated for three times to improve the compression ratio. If repeated for more than three times then there will be increase in time and it may affect other performance factor. Hence it is essential for this algorithm not to be repeated for more than three times.

The first frame is decompressed using $\mathrm{BCH}$ followed by Super-Spatial Structure Prediction decoder. After the reproduction of the first frame the difference of the rest of the frames are decompressed. The first frame becomes the reference frame for the next frame. After the reproduction of the second frame it becomes the reference frame for the next frame and the process continues until all the frames are decompressed.

\section{Results and Discussion}

The proposed methodology has been simulated in Microsoft Visual Studio .Net 2005. To evaluate the performance of the proposed methodology we have tested it on a sequence of MRI and CE images. Medical video is taken from Sundaram Medical Foundation (SMF) and MR-TIP database. Input image sequences are taken from these videos. More than 100 image sequences of MRI and CE are taken and tested for compression. The results are evaluated based on Compression Ratio and Bits per pixel. Figure 2 shows five CE Image sequences and Figure 3 shows five MRI Image Sequences. The images in these CE sequences are of dimension $1024 \times 768$ and the images in these MRI sequences are of dimension $514 \times 514$. Motion Estimation and Motion Compensation is applied to these image sequences using Inverse Diamond Search algorithm and Super-Spatial Structure Compression is applied. The output code of SSP is then taken and BCH is applied to enhance the efficiency of compression ratio. Super-Spatial Structure Prediction significantly reduces the prediction error. SSP outperforms CALIC and saves the bit rate for high frequency image components. With Inverse Diamond Search algorithm the accuracy is $92 \%$ and the time saved is $95 \%$ on the average. Bose,Chaudhuri and Hocquenghem gives a good compression ratio and keeps the time and complexity minimum. 


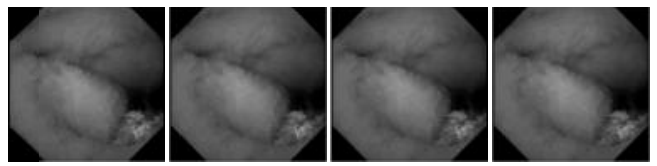

Figure 2. CE Image Sequences
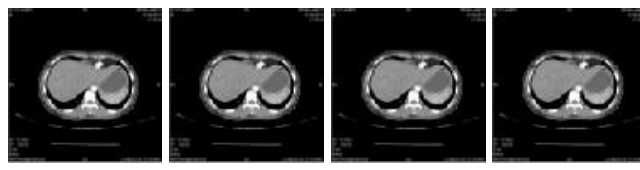

Figure 3. MRI Image Sequences

Table 1 shows the compression ratio of five CE image sequences among the tested image sequences. The results of this proposed methodology are compared with the existing methodology. The result of this algorithm is graphically shown in Figure 4.The results of the proposed algorithm were better.

Table 1. CR of CE Image Sequence

\begin{tabular}{ccc}
\hline $\begin{array}{c}\text { Image } \\
\text { Sequences }\end{array}$ & SSP (Existing) & $\begin{array}{c}\text { Compression Ratio(CR) } \\
\text { SSP+IDS+BCH } \\
\text { (Proposed) }\end{array}$ \\
\hline FI & 5.26 & 6.81 \\
F2 & 5.37 & 6.92 \\
F3 & 5.62 & 7.12 \\
F4 & 5.14 & 6.75 \\
F5 & 5.46 & 7.04 \\
AVG & $\mathbf{5 . 3 7}$ & $\mathbf{6 . 9 3}$ \\
\hline
\end{tabular}

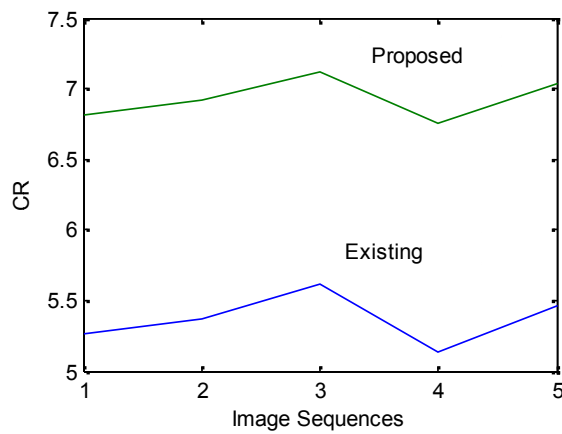

Figure 4. Compression ratio of $\mathrm{CE}$ image sequences

From Table 1 and Figure 4 it is easily identified that the proposed methodology has a better compression ratio than the existing one. On the average the proposed has the compression ratio of 6.93 and existing has 5.67.

Table 2 shows average compression ratio of MRI image sequences among the tested image sequences. The results of this proposed methodology are compared with the existing methodology. The proposed has an average compression ratio of 7.52 which outperforms the other state-of-art algorithm and this is illustrated in Figure 5. Experimental results of the proposed methodology gives 30\% more reduction than the other state-of-the-art algorithms. 
Table 2. Average CR of MRI Image Sequence

\begin{tabular}{cc}
\hline Method & CR \\
\hline JPEG 2000 & 2.596 \\
JPEG-LS & 2.727 \\
JPEG-LS+MV+VAR & 4.841 \\
SSP & 6.25 \\
Proposed & $\mathbf{7 . 7 2}$ \\
\hline
\end{tabular}

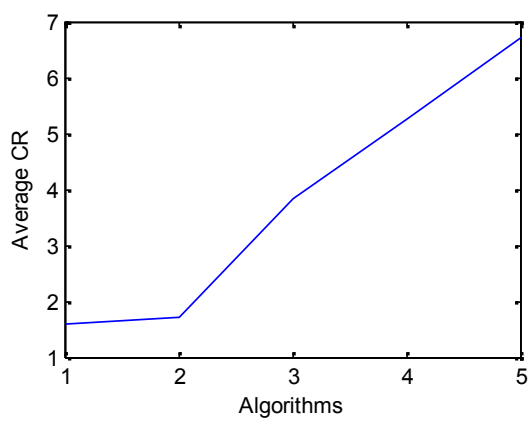

1. JPEG-2000

2. JPEG-LS

3. JPEG-LS+MV+VAR

4. SSP

5. PROPOSED (SSP+IDS+BCH)

Figure 5. Average CR of MRI Image sequences with Existing algorithms

Table 3 shows the Bits per Pixel (BPP) of CE sequences and MRI sequences.

From Table 3 the proposed methodology has 1.503 bpp for CE sequences and 0.82 bpp for MRI sequences. The results show that the proposed methodology produced improved results.

Table 3. Bits per pixel of CE and MRI Image Sequences

\begin{tabular}{ccc}
\hline Method & \multicolumn{2}{c}{ Bits Per Pixel (BPP) } \\
& CE Sequence & $\begin{array}{c}\text { MRI } \\
\text { Sequence }\end{array}$ \\
\hline JPEG 2000 & 2.757 & 3.08 \\
JPEG-LS & 2.394 & 2.93 \\
JPEG-LS+MV+VAR & 2.115 & 1.65 \\
SSP & 1.827 & 1.28 \\
Proposed & $\mathbf{1 . 5 0 3}$ & $\mathbf{0 . 8 2}$ \\
\hline
\end{tabular}

\section{Conclusion}

The algorithm given in this paper makes use of the lossless image compression technique and video compression to achieve higher CR. To achieve high CR the proposed method combines Super-Spatial Structure Prediction (SSP) with inter-frame coding along with Bose, Chaudhuri and Hocquenghem $(\mathrm{BCH})$. The technique used in proposed algorithm gives better result than JPEG-LS and SSP. Fast block-matching algorithm is used here. Since the full search block matching was time consuming as proposed in [1] we have taken Inverse Diamond Search (IDS) algorithm for block-matching process. Inverse Diamond Search (IDS) is faster than Diamond Search (DS) as the number of searches and search points are low. Since this paper exploits inter-frame correlation in the form of MEMC the proposed is compared with [1] [15]. To enhance the compression ratio, SSP is combined with $\mathrm{BCH}$. From Table 1, 2 and 3 it is analyzed that proposed is much better than other state-of-the art lossless compression methods. 


\section{References}

[1] Shaou-Gang Miaou, Fu-Sheng Ke, and Shu-Ching Chen. A Lossless Compression Method for Medical Image Sequences Using JPEG-LS and Interframe Coding. IEEE Transaction on Information Technology in Biomedicine. 2009; 13(5).

[2] GM Padmaja, P Nirupama. Analysis of Various Image Compression Techniques. ARPN Journal of Science and Technology. 2012; 2(4).

[3] Ansam Ennaciri, Mohammed Erritali, Mustapha Mabrouki, Jamaa Bengourram. Comparative Study of Wavelet Image Compression: JPEG2000 Standart. TELKOMNIKA Indonesian Journal of Electrical Engineering. 2015; 16(1).

[4] SE Ghare, MA Mohd Ali, K Jumari and M Ismail. An Efficient Low Complexity Lossless Coding Algorithm for Medical Images. American Journal of Applied Sciences. 2009; 6 (8): 1502-1508.

[5] Arikatla Hazarathaiah, B Prabhakara Rao. Medical Image Compression using Lifting based New Wavelet Transforms. International Journal of Electrical and Computer Engineering (IJECE). 2014; 4(5): 741-750.

[6] S Bhavani, Dr K Thanushkodi. A Survey in Coding Algorithms in Medical Image Compression. International Journal on Computer Science and Engineering. 2010; 02(5): 1429-1434.

[7] Xiwen Owen Zhao, Zhi hai Henry He. Lossless Image Compression Using Super-Spatial Structure Prediction. IEEE Signal Processing. 2010; 17(4).

[8] CS Rawat, Seema G Bhatea, Dr Sukadev Meher. A Novel Algoritm of Super-Spatial Structure Prediction for RGB Colourspace. International Journal of Scientific \& Engineering Research. 2012; $3(2)$.

[9] X Wu and N Memon. Context-based, adaptive, lossless image coding. IEEE Trans.Commun. 1997; 45(4): 437-444.

[10] X Wu. Lossless Compression of Continuous-tone Images via Context Selection, Quantization, and Modeling. IEEE Trans. Image Processing. 1997; 6(5): 656-664.

[11] YD Wang. The Implementation of Undistorted Dynamic Compression Technique for Biomedical Image. Master's thesis. Dept. Electr. Eng; Nat.Cheng Kung Univ; Taiwan. 2005.

[12] D Brunello, G Calvagno, GA Mian and R Rinaldo. Lossless Compression of Video using Temporal Information. IEEE Trans. Image Process. 2003; 12(2): 132-139.

[13] ND Memon and Khalid Sayood. Lossless Compression of Video Sequences. IEEE Trans. Commun. 44(10): 1340-1345.

[14] MF Zhang, J Hu, and LM Zhang. Lossless Video Compression using Combination of Temporal and Spatial Prediction. In Proc. IEEE Int. Conf. Neural Newt. Signal Process. 2003; 2: 1193-1196.

[15] Mudassar Raza, Ahmed Adnan, Muhammad Sharif and Syed Waqas Haider. Lossless Compression Method for Medical Image Sequences Using Super-Spatial Structure Prediction and Inter-frame Coding. International Journal of Advanced TResearch and Technology. 2012; 10(4).

[16] Wen-Jan Chen and Hui-Min Chen. Inverse Diamond Search Algorithm for 3D Medical Image Set Compression. Journal of Medical and Biological Engineering. 2009; 29(5): 266-270.

[17] A Alarabeyyat, S Al-Hashemi, T Khdour, M Hjouj Btoush, S Bani-Ahmad and R Al-Hashemi. Lossless Image Compression Technique Using Combination Methods. Journal of Software Engineering and Applications. 2012; 5: 752-763.

[18] T Wiegand, GJ Sullivan, G Bjntegaard and A Luthra. Overview of the H.264/AVC video coding standard. IEEE Trans. Circuits Systems Video Technology. 2003; 13(7). 